\title{
SKEIN ALGEBRA OF A GROUP
}

\author{
JÓZEF H. PRZYTYCKI \\ Department of Mathematics, The George Washington University \\ 2201 G Str., room 428, Funger Hall, Washington, D.C. 20052, U.S.A. \\ E-mail: przytyck@math.gwu.edu \\ ADAM S. SIKORA \\ Department of Mathematics, Warsaw University, ul. Banacha 2, 02-097 Warszawa, Poland \\ E-mail: asikora@math.umd.edu
}

We define for each group $G$ the skein algebra of $G$. We show how it is related to the Kauffman bracket skein modules. We prove that skein algebras of abelian groups are isomorphic to symmetric subalgebras of corresponding group rings. Moreover, we show that, for any abelian group $G$, homomorphisms from the skein algebra of $G$ to $C$ correspond exactly to traces of $S L(2, C)$-representations of $G$. We also solve, for abelian groups, the conjecture of Bullock on $S L(2, C)$ character varieties of groups - we show that skein algebras are isomorphic to the coordinate rings of the corresponding character varieties.

\section{Definition of the skein module and of the skein algebra of a group}

Definition 1.1 ([Pr, H-P-1]). Let $M$ be any 3-manifold and let $\mathcal{L}^{f r}$ denote the set of ambient isotopy classes of framed unoriented links in $M$ (including the empty link $\emptyset$ ). Let $R$ be any commutative ring with 1 and $A$ an invertible element in $R$. Furthermore, let $S_{2, \infty}$ be the submodule of $R \mathcal{L}^{f r}$ generated by skein expressions $L_{+}-A L_{0}-A^{-1} L_{\infty}$ and $L \amalg \bigcirc+\left(A^{2}+A^{-2}\right) \cdot L$, where the triple $L_{+}, L_{0}, L_{\infty}$ is presented in Fig. 1.1 (that is, $L_{+}, L_{0}, L_{\infty}$ are three framed links which are the same outside a small oriented 3ball but inside the ball they are as in Fig. 1.1. If $M$ is oriented then the orientation of the ball agrees with that of $M ; L \amalg \bigcirc$ denotes a disjoint union of a link $L$ with a trivial component $(\bigcirc)$ ). We define the Kauffman bracket skein module as the quotient $\mathcal{S}_{2, \infty}(M ; R, A)=R \mathcal{L}^{f r} / S_{2, \infty}$. If $R=Z\left[A^{ \pm 1}\right]$, we write briefly $\mathcal{S}_{2, \infty}(M)$.

1991 Mathematics Subject Classification: 57M, $20 \mathrm{C} 07$.

The paper is in final form and no version of it will be published elsewhere. 


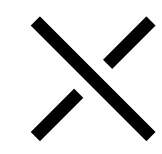

$\mathrm{L}_{+}$

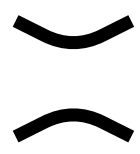

$\mathrm{L}_{0}$

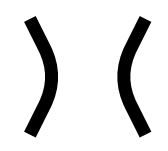

$\mathrm{L}_{\infty}$

Fig. 1.1

The above definition determines a functor from the category of 3-manifolds and embeddings (preserving orientation in the case of oriented manifolds) to the category of $R$-modules (with a specified invertible element $A \in R$ ). In particular if $f: M \rightarrow N$ is a manifold embedding then $f_{*}: \mathcal{S}_{2, \infty}(M ; R, A) \rightarrow \mathcal{S}_{2, \infty}(N ; R, A)$ denotes the associated homomorphism of modules.

We show that if we put $A=-1$ then $\mathcal{S}_{2, \infty}(M ; R)_{(A=-1)}$ becomes a commutative algebra depending only on the fundamental group of the manifold (Lemma 1.3). The product in $\mathcal{S}_{2, \infty}(M ; R)_{(A=-1)}$ is given by a disjoint sum of links and the identity is the empty link. Motivated by this, we define the skein algebra of any group as follows.

DeFinition 1.2. Let $G$ be any group (with identity denoted by $e$ ), $R$ a commutative ring with 1 and $R G$ the group algebra over $G$ with coefficients in $R$. Let $\mathbf{T} R G$ be the tensor algebra over the module $R G$ (with the identity denoted by 1 ). Let $\mathcal{I}^{+}$be the ideal of $\mathbf{T} R G$ generated by $e+2$ and expressions $g \otimes h-h \otimes g, g \otimes h+g h+g h^{-1}$, for any $g, h \in G$. We define the skein algebra of $G$ as $\mathcal{S}^{+}(G ; R)=\mathbf{T} R G / \mathcal{I}^{+}$.

We list below some elementary properties of $\mathcal{S}^{+}(G ; R)$.

(0) $\mathcal{S}^{+}(-; R)$ yields a functor from the category of groups to the category of $R$ algebras. This functor sends epimorphisms of groups to epimorphisms of algebras ${ }^{1}$.

(1) For any $g \in G, g \otimes e=-g e-g e^{-1}=-2 g$. This has motivated us to put $e=-2$, so we have generally $w \otimes e=-2 w$.

(2) $g=g^{-1}$, because $-2 g=e \otimes g=-g-g^{-1}$.

(3) $h g h^{-1}=g$, because

$$
\begin{aligned}
0=g \otimes h-h \otimes g & =-g h-g h^{-1}+h g+h g^{-1} \\
& =-g h+h g-g h^{-1}+\left(g h^{-1}\right)^{-1}=-g h+h g .
\end{aligned}
$$

Thus $g h=h g$ and, finally, $(h g) h^{-1}=h^{-1}(h g)=g$.

(4) The commutator $[g, h]=g h g^{-1} h^{-1}$ satisfies the equality

$$
-[g, h]=g \otimes g+h \otimes h+g h \otimes g h+g h \otimes g \otimes h-2 .
$$

Proof. $-g h g^{-1} h^{-1}=g h g^{-1} \otimes h^{-1}+g h g^{-1} h=h \otimes h-\left(g h \otimes g^{-1} h+g h h^{-1} g\right)=$ $h \otimes h-g h \otimes g h^{-1}-g g=h \otimes h-g h \otimes g h^{-1}+g \otimes g+g g^{-1}=g \otimes g+h \otimes h-g h \otimes g h^{-1}+e=$ $g \otimes g+h \otimes h-g h \otimes(-g \otimes h-g h)-2=g \otimes g+h \otimes h+g h \otimes g h+g h \otimes g \otimes h-2$.

${ }^{1}$ For monomorphisms the analogous fact does not hold. For example, if we consider an embedding $i: Z_{3} \rightarrow S_{3}$, then for $i_{*}: \mathcal{S}^{+}\left(Z_{3} ; C\right) \rightarrow \mathcal{S}^{+}\left(S_{3} ; C\right)$ one has $i_{*}(g+2)=0$ for any $g \in Z_{3}$. Moreover, skein algebras can be isomorphic even if groups are not isomorphic. For example, an embedding $j: Z_{2} \rightarrow S_{3}$ yields an isomorphism $j_{*}: \mathcal{S}^{+}\left(Z_{2} ; C\right) \rightarrow \mathcal{S}^{+}\left(S_{3} ; C\right)$; [P-S-2]. 
(5) (Universal coefficients property). Let $r: R \rightarrow R^{\prime}$ be a homomorphism of rings (commutative with 1). We can think of $R^{\prime}$ as an $R$-module. Then the identity map on $G$ induces isomorphism of $R^{\prime}$-algebras:

$$
\mathcal{S}^{+}\left(G ; R^{\prime}\right) \simeq \mathcal{S}^{+}(G ; R) \otimes_{R} R^{\prime}
$$

In particular $\mathcal{S}^{+}(G ; R) \simeq \mathcal{S}^{+}(G ; Z) \otimes_{Z} R$. The analogous universal coefficients property holds also for skein modules $[\operatorname{Pr}]($ Lemma 5$)$.

Proof. We use the "five lemma" to show that the algebra homomorphism

$$
u: \mathcal{S}^{+}(G ; R) \otimes_{R} R^{\prime} \rightarrow \mathcal{S}^{+}\left(G ; R^{\prime}\right),
$$

$u(g)=g$ is an $R$ - (and $R^{\prime}$-) isomorphism. Namely, the exact sequence of $R$ modules

$$
\mathcal{I}^{+}(R) \rightarrow \mathbf{T} R G \rightarrow \mathcal{S}^{+}(G ; R) \rightarrow 0
$$

leads to the exact sequence

$$
\mathcal{I}^{+}(R) \otimes_{R} R^{\prime} \rightarrow \mathbf{T} R G \otimes_{R} R^{\prime} \rightarrow \mathcal{S}^{+}(G ; R) \otimes_{R} R^{\prime} \rightarrow 0 .
$$

Now, using the "five lemma" to the commutative diagram (with exact rows)

$$
\begin{aligned}
& \mathcal{I}^{+}(R) \otimes_{R} R^{\prime} \rightarrow \mathbf{T} R G \otimes_{R} R^{\prime} \rightarrow \mathcal{S}^{+}(G ; R) \otimes_{R} R^{\prime} \rightarrow 0 \\
& \downarrow \text { epi } \quad \downarrow \text { iso } \quad \downarrow u \\
& \mathcal{I}^{+}\left(R^{\prime}\right) \quad \rightarrow \quad \mathrm{T} R^{\prime} G \quad \rightarrow \quad \mathcal{S}^{+}\left(G ; R^{\prime}\right) \quad \rightarrow 0
\end{aligned}
$$

we conclude that $u$ is an isomorphism of $R^{\prime}$ - (and $R$-) modules, thus also of algebras.

We will use the above properties of skein algebras to show that for a 3-manifold and $A=-1$ the skein module (algebra) $\mathcal{S}_{2, \infty}(M ; R,-1)$ is naturally isomorphic to the skein algebra of the fundamental group of $M, \mathcal{S}^{+}\left(\pi_{1}(M) ; R\right)$.

LEMMA 1.3 (Basic Lemma). Let $M$ be a connected 3-manifold, $R$ a commutative ring with identity and $A=-1$. Then:

(a) $\mathcal{S}_{2, \infty}(M ; R,-1)$ is a commutative algebra with a product given by a disjoint sum of links.

(b) Consider a function $\xi$ from the space $\mathcal{K}^{\text {fr }}$ of framed unoriented knots in $M$ to $\mathcal{S}^{+}\left(\pi_{1}(M) ; R\right)$, where $\xi(K)$ belongs to the conjugacy class of an element of $\pi_{1}(M)$ yielded by $K$ (for some orientation of $K$ ) and $\xi(\emptyset)=1$. Then $\xi$ descends to an $R$-algebra isomorphism

$$
\widehat{\xi}: \mathcal{S}_{2, \infty}(M ; R,-1) \rightarrow \mathcal{S}^{+}\left(\pi_{1}(M) ; R\right)
$$

Proof. (a) Notice that if $A=-1$, then $L_{+}=-L_{0}-L_{\infty}=L_{-}$in $\mathcal{S}_{2, \infty}(M ; R, A)$ (see Fig. 1.2) and $L_{1} \cdot L_{2}$ does not depend on the relative position of $L_{1}$ with respect to $L_{2}^{2}$. In particular $L_{1} \cdot L_{2}=L_{2} \cdot L_{1}$.

\footnotetext{
${ }^{2}$ We use the standard fact that two embeddings of a compact graph in $M$ are homotopic iff one can be obtained from the other by crossing changes and an ambient isotopy.
} 


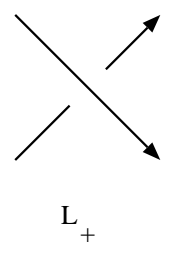

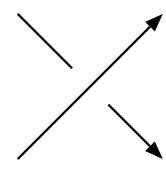

L

Fig. 1.2

(b) Properties (2) and (3) of skein algebras imply that $\xi$ is well defined. Because $\mathcal{S}^{+}\left(\pi_{1}(M) ; R\right)$ is commutative, we can extend $\xi$ to the space of links in $M, \mathcal{L}^{f r}$, by the rule $\xi(L)=\xi\left(K_{1}\right) \otimes \xi\left(K_{2}\right) \otimes \ldots \otimes \xi\left(K_{n}\right)$, where the link $L$ has components $K_{1}, K_{2}, \ldots, K_{n}$. Then we extend $\xi$ linearly from $\mathcal{L}^{f r}$ to $R \mathcal{L}^{f r}$. Since

$$
\xi(\bigcirc)=-2 \text { and } \xi\left(K_{+}\right)+\xi\left(K_{0}\right)+\xi\left(K_{\infty}\right)=0,
$$

$\xi$ descends to an $R$-algebra epimorphism $\widehat{\xi}: \mathcal{S}_{2, \infty}(M ; R,-1) \rightarrow \mathcal{S}^{+}\left(\pi_{1}(M) ; R\right)$. To see that $\widehat{\xi}$ is a monomorphism it suffices to construct the inverse map $\widehat{\xi}^{-1}$. We start the construction by introducing the homomorphism $\mu: \mathbf{T} R \pi_{1}(M) \rightarrow \mathcal{S}_{2, \infty}(M ; R,-1)$ which associates to any element $x$ of $\pi_{1}(M)$ a knot $K_{x}$ which represents it. It is a well defined map because, as observed in (a), homotopic knots yield the same element in $\mathcal{S}_{2, \infty}(M ; R,-1)$, in particular the condition $A=-1$ makes knots independent on framing.

$\mu$ descends to the homomorphism $\widehat{\mu}: \mathcal{S}^{+}\left(\pi_{1}(M) ; R\right) \rightarrow \mathcal{S}_{2, \infty}(M ; R,-1)$ because $\mu$ sends the ideal $\mathcal{I}^{+}$to zero:

(i) $\mu(x \otimes y-y \otimes x)=K_{x} K_{y}-K_{y} K_{x}=0$ (part (a)).

(ii) $\mu(e+2)=\left(-A^{2}-A^{-2}\right) \emptyset+2 \emptyset=0$ (for $\left.A=-1\right)$.

(iii) $\mu\left(x \otimes y+x y+x y^{-1}\right)=K_{x} \cdot K_{y}+K_{x y}+K_{x y^{-1}}=K_{+}+K_{0}+K_{\infty}=0$ (skein relation).

$\widehat{\mu}$ is the inverse of $\widehat{\xi}$. Thus the proof of the Basic Lemma is complete.

It is convenient to consider a variant of the skein algebra of a group (isomorphic to the previous one):

Definition 1.4. Let $\mathbf{T} R G$ be, as in Definition 1.2, the tensor algebra over the module $R G$. Let $\mathcal{I}^{-}$be the ideal of $\mathbf{T} R G$ generated by expressions $g \otimes h-h \otimes g, g \otimes h-g h-g h^{-1}$ and $e-2$. We define the algebra $\mathcal{S}^{-}(G ; R)$ as $\mathbf{T} R G / \mathcal{I}^{-}$.

Lemma 1.5. The R-algebra homomorphism $\widehat{\beta}: \mathcal{S}^{+}(G ; R) \rightarrow \mathcal{S}^{-}(G ; R)$, given by $\widehat{\beta}(g)=-g$ for $g \in G$, is an isomorphism of $R$-algebras.

Proof. We use the fact that the isomorphism $\beta: \mathbf{T} R G \rightarrow \mathbf{T} R G, \beta(g)=-g$, sends $\mathcal{I}^{+}$to $\mathcal{I}^{-}$. Therefore $\mathbf{T} R G / \mathcal{I}^{+} \rightarrow \mathbf{T} R G / \mathcal{I}^{-}$is an isomorphism.

2. The skein algebra of an abelian group. We start by introducing two subalgebras of the group algebra $R G$.

Definition 2.1. (i) $\operatorname{sym}(R G)$ is the subalgebra of $R G$ generated by elements of the form $g+g^{-1}$, for $g \in G$. 
(ii) $R G^{\text {sym }}$ is the subalgebra of $R G$ composed of elements invariant under the involution (anti-isomorphism) $\tau: R G \rightarrow R G$, given by $\tau(g)=g^{-1}$, for $g \in G$.

LEMMA 2.2. Let $G$ be an abelian group. Then

(i) $\operatorname{sym}(R G)$ is a free $R$-module with a basis $\{e\} \cup\left\{g+g^{-1}\right\}_{g \in B}$, where

(a) $B=G-\{e\}$, if $2 \neq 0$ in $R$,

(b) $B=\left\{g \in G: g^{2} \neq e\right\}$, if $2=0$ in $R$.

(ii) $R G^{\text {sym }}$ is a free $R$-module with a basis composed of elements $g+g^{-1}$, where $g^{2} \neq e$, and elements $g$, for $g^{2}=e$.

(iii) $\operatorname{sym}(R G)=R G^{\text {sym }}$ if and only if either 2 is invertible in $R$ or $G$ has no nontrivial element of order two.

Proof. (i) Since $\left(g+g^{-1}\right)\left(h+h^{-1}\right)=g h+(g h)^{-1}+g h^{-1}+\left(g h^{-1}\right)^{-1}, \operatorname{sym}(R G)$ is generated as an $R$-module by $\{e\}$ and elements $\left\{g+g^{-1}\right\}$, for $g \in G$. Because elements of $G$ form a basis of $R G$, the set of all nonzero elements of the form $g+g^{-1}$ together with the element $e$ is $R$-linearly independent in $R G$. Hence, if $2 \neq 0$ in $R$, then $\left\{g+g^{-1}\right.$ : $g \in G \backslash\{e\}\} \cup\{e\}$ is a basis of $\operatorname{sym}(R G)$. If $2=0$ in $R$, then $g+g^{-1}=0$ iff $g^{2}=e$. Therefore, in that case $\left\{g+g^{-1}: g \in G, g^{2} \neq e\right\} \cup\{e\}$ is a basis of $\operatorname{sym}(R G)$.

(ii) Let $w \in R G^{s y m}$. Then $w=\sum_{b \in B} a_{b} b+\sum_{g \in A} a_{g} g$, where $B=\left\{g+g^{-1}: g \in\right.$ $\left.G, g^{2} \neq e\right\}, A=\left\{g: g \in G, g^{2}=e\right\}$ and $a_{b}, a_{g} \in R$. Thus elements listed in (ii) generate $R G^{\text {sym }}$. We can argue as in (i) that they are linearly independent.

(iii) It follows immediately from (i) and (ii).

THEOREM 2.3. For any abelian group $G$ consider the algebra homomorphism $\phi$ : $\mathbf{T} R G \rightarrow R G$ given by $\phi(g)=g+g^{-1}$ for any $g \in G$. Then $\phi$ descends to the algebra homomorphism $\widehat{\phi}: \mathcal{S}^{-}(G ; R) \rightarrow R G$. Furthermore, if $2 \neq 0$ in $R$ or $G$ has no nontrivial elements of order 2 , then $\widehat{\phi}$ is a monomorphism (in particular $\mathcal{S}^{-}(G ; R$ ) is isomorphic to $\operatorname{sym}(R G))$.

Proof. (1) $\widehat{\phi}$ is well defined, because $\phi(g) \phi(h)=\phi(h) \phi(g)$ and $\phi\left(g \otimes h-g h-g h^{-1}\right)=$ $\phi(g) \phi(h)-\phi(g h)-\phi\left(g h^{-1}\right)=\left(g+g^{-1}\right)\left(h+h^{-1}\right)-\left(g h+(g h)^{-1}\right)-\left(g h^{-1}+g^{-1} h\right)=0$

(2) $\widehat{\phi}$ is a monomorphism:

(i) From the degree reducing identity, $g \otimes h=g h+g h^{-1}$, it follows that $\left(G / g \sim g^{-1}-\right.$ $\{e\}) \cup\{1\}$ is a generating set of the $R$-module $\mathcal{S}^{-}(G ; R)$.

(ii) By the definition, $\phi(\mathbf{T} R G)=\operatorname{sym}(R G)$. From Lemma 2.2 it follows that the expressions $g+g^{-1}$ for $g \in(G-\{e\})$ and $\{e\}$ form a basis for the module $\operatorname{sym}(R G)$.

(iii) $\widehat{\phi}$ sends bijectively a generating set of the $R$-module $\mathcal{S}^{-}(G ; R)$ to a basis of $\operatorname{sym}(R G)$ thus $\widehat{\phi}: \mathcal{S}^{-}(G ; R) \rightarrow \operatorname{sym}(R G)$ is a module isomorphism (and hence an algebra isomorphism).

Corollary 2.4. Let $G$ be an abelian group. Then $\phi(g)=\phi(h)$ if and only if

(i) $g=h^{ \pm 1}$, or

(ii) $2=0$ in $R$ and $g^{2}=h^{2}=e$ in $G$. 
COROLlary 2.5. (a) $\mathcal{S}_{2, \infty}(L(p, q) ; R,-1) \simeq \operatorname{sym}\left(R Z_{p}\right)^{3}$. This result agrees with the module structure of the general skein module $\mathcal{S}_{2, \infty}(L(p, q) ; R, A)$ given in $[\mathrm{H}-\mathrm{P}-2, \mathrm{H}-\mathrm{P}-3]{ }^{4}$.

(b) $\mathcal{S}_{2, \infty}\left(T^{2} \times[0,1] ; R,-1\right) \simeq \operatorname{sym}(R(Z \oplus Z))^{5}$. This is the special case of the general computation of the Kauffman bracket skein algebra of $T^{2} \times[0,1][\mathrm{B}-\mathrm{P}]$.

(c) $\mathcal{S}_{2, \infty}\left(T^{3} ; R,-1\right) \simeq \operatorname{sym}(R(Z \oplus Z \oplus Z))$. (The structure of the general skein module $\mathcal{S}_{2, \infty}\left(T^{3}\right)$ is still unknown and is more complicated than in the case of $A=-1$; in particular the skein module has a torsion part).

THEOREM 2.6. If $i: G_{1} \rightarrow G_{2}$ is a monomorphism and $G_{2}$ is abelian then $i_{*}$ : $\mathcal{S}^{-}\left(G_{1} ; R\right) \rightarrow \mathcal{S}^{-}\left(G_{2} ; R\right)$ is a monomorphism for any ring $R$.

Proof. Lemma 2.2 and Theorem 2.3 imply that $\mathcal{S}^{+}\left(G_{i} ; Z\right), i=1,2$, is a free $Z$ module with a basis $\{e\} \cup\left\{g+g^{-1}: g \in G_{i}, g \neq e\right\}$. The inclusion $i: G_{1} \rightarrow G_{2}$ yields a homomorphism of skein algebras $i_{*}: \mathcal{S}^{-}\left(G_{1} ; Z\right) \rightarrow \mathcal{S}^{-}\left(G_{2} ; Z\right)$ carrying the elements of the basis of $\mathcal{S}^{-}\left(G_{1} ; Z\right)$ to the elements of the basis of $\mathcal{S}^{-}\left(G_{2} ; Z\right)$. By universal coefficients property, $\mathcal{S}^{-}\left(G_{i} ; R\right)=\mathcal{S}^{-}\left(G_{i} ; Z\right) \otimes R$. Hence the induced homomorphism $i_{*}^{\prime}: \mathcal{S}^{-}\left(G_{1} ; R\right) \rightarrow \mathcal{S}^{-}\left(G_{2} ; R\right)$ also carries the elements of the basis of $\mathcal{S}^{-}\left(G_{1} ; R\right)$ to the elements of the basis of $\mathcal{S}^{-}\left(G_{2} ; R\right)$. Therefore $i_{*}^{\prime}$ is a monomorphism.

3. Character variety of a group. Let $\varrho: G \rightarrow S l_{2}(C)$ be a representation of a group $G$ in $S l_{2}(C)$. Then the trace of this representation $\chi(g)=\operatorname{tr} \varrho(g), \chi: G \rightarrow C$, is called briefly a character of $G$. We denote the set of all characters of $G$ by $X(G)$; this notation agrees with that of Culler and Shalen in [C-S], provided that $G$ is finitely generated.

In the remainder of this paper we are going to focus on the relationship between $\mathcal{S}^{-}(G ; C)$ and $X(G)^{6}$.

Let $\chi$ be a character of $G$. Then $\chi: G \rightarrow C$ can be extended to the homomorphism of $C$-algebras $\bar{\chi}: \mathbf{T} C G \rightarrow C$ such that

$$
\bar{\chi}(a \otimes b)=\bar{\chi}(a) \cdot \bar{\chi}(b), \quad \bar{\chi}(a+b)=\bar{\chi}(a)+\bar{\chi}(b),
$$

and

$$
\bar{\chi}(g)=\chi(g)
$$

for any $a, b \in \mathbf{T} C G, g \in G$. It can easily be seen that $\bar{\chi}(e-2)=0$ and $\bar{\chi}(g \otimes h-h \otimes g)=0$ for $g, h \in G$. Moreover, the well known equality (of H. Vogt, and R. Fricke and F. Klein;

\footnotetext{
${ }^{3}$ One can show further that the algebra is isomorphic to $R[y] / I_{p}$, where $I_{0}=\{0\}$, and otherwise $I_{p}$ is the ideal generated by the polynomial $Q_{[p / 2]+1}(y)-Q_{p-[p / 2]-1}(y)$, where $[x]$ is the integer part of $x . Q_{n}$ is the Chebyshev polynomial of the first kind, $Q_{n}(y)=y Q_{n-1}(y)-Q_{n-2}(y)$, $Q_{0}(y)=2, Q_{1}(y)=y$; [P-S-2].

${ }^{4}$ In fact for the projective space, $L(2,1), \mathcal{S}_{2, \infty}(L(2,1) ; R, A)$ has an algebra structure and as an algebra it is isomorphic to $R[\alpha] /\left(\alpha^{2}-A^{3} \frac{A^{4}-A^{-4}}{A-A^{-1}}\right)$, which implies $\mathcal{S}^{+}\left(Z_{2} ; R\right)=R[\alpha] /\left(\alpha^{2}-4\right)$ for $A=-1$.

${ }^{5}$ One can show further that the algebra is isomorphic to $R[x, y, z] /\left(x^{2}+y^{2}+z^{2}-x y z\right.$ -4) [B-P, P-S-1].

${ }^{6} \mathrm{D}$. Bullock was the first to investigate the relation between the Kauffman bracket skein module of a 3-manifold, $M$, and $X\left(\pi_{1}(M)\right)$ [B-1, B-2].
} 
compare [C-S, Jo, L-M, Ma-1])

$$
\operatorname{tr}(a) \operatorname{tr}(b)=\operatorname{tr}(a b)+\operatorname{tr}\left(a b^{-1}\right)
$$

for $a, b \in S l_{2}(C)$ implies that

$$
\bar{\chi}(g \otimes h)=\bar{\chi}(g h)+\bar{\chi}\left(g h^{-1}\right)
$$

for $g, h \in G$. Therefore $\mathcal{I}^{-} \subset K e r \bar{\chi}$ and $\bar{\chi}$ induces a homomorphism

$$
h_{\chi}: \mathcal{S}^{-}(G ; C)=\mathbf{T} C G / \mathcal{I}^{-} \rightarrow C,
$$

such that $h_{\chi}([g])=\bar{\chi}(g)=\chi(g)$ for $g \in G$. Hence for any group $G$ and for any character $\chi \in X(G)$ the following diagram commutes:

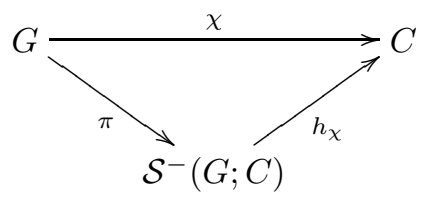

where $\pi(g)=[g] \in \mathcal{S}^{-}(G ; C)$ for $g \in G$.

THEOREM 3.1. If $G$ is an abelian group then each homomorphism of algebras $h$ : $\mathcal{S}^{-}(G ; C) \rightarrow C$ is yielded by a character of $G$, i.e. $h=h_{\chi}$ for some $\chi \in X(G)$.

Proof. By Theorem 2.3, $\widehat{\phi}: \mathcal{S}^{-}(G, C) \rightarrow \operatorname{sym}(C G), \widehat{\phi}(g)=g+g^{-1}$ for $g \in G$, is an isomorphism of $C$-algebras. The ring $C G$ (resp. $\operatorname{sym}(C G)$ ) is generated by elements of the form $\frac{g \pm g^{-1}}{2}$ (resp: $\left.\frac{g+g^{-1}}{2}\right)$. Since $\left(\frac{g-g^{-1}}{2}\right)^{2}=\left(\frac{g+g^{-1}}{2}\right)^{2}-1, C G$ is an integral extension of $\operatorname{sym}(C G)$. Let us consider any $C$-algebra homomorphism $h: \mathcal{S}^{-}(G, C) \rightarrow C$ and the composition $h \circ \widehat{\phi}^{-1}: \operatorname{sym}(C G) \rightarrow C$. The kernel of $h \circ \widehat{\phi}^{-1}$ is a maximal ideal in $\operatorname{sym}(C G)$ and therefore, by Lying-over Theorem, it can be extended to a maximal ideal $J \triangleleft C G$ (see e.g. [Hu] Ch. VIII). Let $f$ be a natural projection $f: C G \rightarrow C G / J=C$. Then the following diagram commutes:

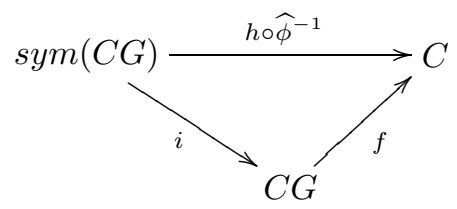

where $i: \operatorname{sym}(C G) \rightarrow C G$ denotes the natural inclusion.

The homomorphism $f$ restricted to $G$ yields a representation of $G$ in the multiplicative group $C^{*}, f_{\mid G}: G \rightarrow C^{*}$. Therefore the function $\chi: G \rightarrow C, \chi(g)=f\left(g+g^{-1}\right)$ is the character of the representation $\varrho: G \rightarrow S l_{2}(C)$

$$
\varrho(g)=\left(\begin{array}{cc}
f(g) & 0 \\
0 & f(g)^{-1}
\end{array}\right) .
$$

We will complete the proof by showing that $h=h_{\chi}$. Let $g \in G$. Then $h_{\chi}([g])=\chi(g)=$ $f\left(g+g^{-1}\right)$. Since $g+g^{-1} \in \operatorname{sym}(C G) \subset C G, f\left(g+g^{-1}\right)=h \circ \widehat{\phi}^{-1}\left(g+g^{-1}\right)=h([g])$.

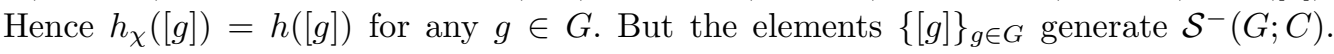
Therefore $h_{\chi}=h$. 
The above proof implies that any character $\chi$ of an abelian group $G$ is yielded by a representation of $G$ into the subgroup of diagonal matrices in $S L_{2}(C)$.

Let $G$ be a finitely generated group. Vogt 1889 [V] and Fricke and Klein (1897)[F-K] stated and Horowitz [Ho] proved that each character $\chi$ of $G$ is uniquely determined by the values $\chi\left(g_{1}\right), \ldots, \chi\left(g_{n}\right)$ for some properly chosen finite subset $\left\{g_{1}, \ldots, g_{n}\right\}$ of $G$ (compare [C-S] and [Ma-2]). Therefore $X(G)$ can be identified with the set

$$
\left\{\left(\chi\left(g_{1}\right), \ldots, \chi\left(g_{n}\right)\right): \chi \text { is a character of } G\right\} \subset C^{n} .
$$

Culler and Shalen proved that $X(G)$ under this identification is an algebraic set and that the definition of $X(G)$ does not depend (up to isomorphism of algebraic sets) on the choice of the elements $g_{1}, \ldots, g_{n}$. They also showed that for any $g \in G$ the function $\tau_{g}(\chi)=\chi(g), \tau_{g}: X(G) \rightarrow C$, is regular on $X(G)$.

One can easily check that $\tau_{e}=2, \tau_{g h}=\tau_{h g}$ and $\tau_{g} \cdot \tau_{h}=\tau_{g h}+\tau_{g h^{-1}}$ for $g, h \in G$. Therefore there exists a homomorphism from $\mathcal{S}^{-}(G ; C)$ to the coordinate ring of $X(G)$

$$
\psi: \mathcal{S}^{-}(G ; C) \rightarrow C[X(G)]
$$

such that $\psi([g])=\tau_{g}$ for $g \in G$. Since $C[X(G)]$ is generated by $\tau_{g_{1}}, \ldots, \tau_{g_{n}}, \psi$ is an epimorphism.

Lemma 3.2. For any $\chi \in X(G)$ we denote the maximal ideal in $C[X(G)]$ corresponding to $\chi$ by $m_{\chi}$, i.e. $m_{\chi}$ is the ideal consisting of all regular functions on $X(G)$ vanishing at $\chi$. Then the following diagram commutes:

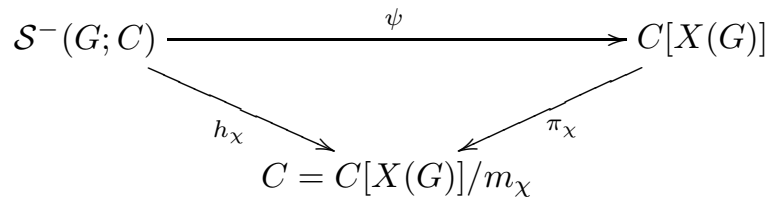

where $\pi_{\chi}$ denotes the natural projection $C[X(G)] \rightarrow C[X(G)] / m_{\chi}$.

Proof. Since $\mathcal{S}^{-}(G ; C)$ is generated by elements $[g]$, where $g \in G$, it suffices to prove that $\pi_{\chi} \psi([g])=h_{\chi}([g])$. But $\pi_{\chi} \psi([g])=\pi_{\chi}\left(\tau_{g}\right)=\chi(g)=h_{\chi}([g])$.

THEOREM 3.3. $\psi: \mathcal{S}^{-}(G ; C) \rightarrow C[X(G)]$ is an isomorphism for any finitely generated abelian group $G$.

Proof. Notice that if $G$ is finitely generated abelian group then $G$ is isomorphic to $T \oplus Z^{k}$, where $T$ is a finite group and $k$ is a non-negative integer. By Maschke's Theorem [see $[\mathrm{Hu}] \mathrm{Ch}$. IX], $C T$ has a trivial Jacobson radical (i.e. $C T$ is semisimple), and therefore $C T$ does not have any non-zero nilpotent elements; (we use here the fact that the Jacobson radical of a commutative algebra contains all nilpotent elements). Since $C T$ is a finitely generated commutative $C$-algebra without non-zero nilpotent elements, $C T$ is a coordinate ring of some algebraic set $A ; C T \simeq C[A]^{7}$.

The algebra $C Z^{k}$ is isomorphic to $C\left[x_{1}^{ \pm 1}, x_{2}^{ \pm 1}, \ldots, x_{k}^{ \pm 1}\right]$ and so, like $C T$, it does not have any nilpotent elements. Therefore we can assume that $C Z^{k}$ is a coordinate ring of

\footnotetext{
${ }^{7}$ In fact, $A$ is a finite set.
} 
an algebraic set $B$ (In fact $B \simeq C^{* k}{ }^{8}$ ). Then $C G=C T \otimes C Z^{k}$ is the coordinate ring of $A \times B$.

Let $s \in \mathcal{S}^{-}(G ; C), s \neq 0$. Then $\widehat{\phi}(s) \neq 0$ in $\operatorname{sym}(C G) \subset C G$. Since $C G \simeq C[A \times B]$, we can consider $\widehat{\phi}(s)$ as a regular function on $A \times B$. Let $m_{a}$ be the maximal ideal in $C[A \times B]$ associated to a point $a \in A \times B$ at which $\widehat{\phi}(s)$ does not vanish. Then the natural homomorphism $h: C[A \times B] \rightarrow C[A \times B] / m_{a} \simeq C$ does not vanish on $\widehat{\phi}(s)$. By Theorem 3.1 , the composition of homomorphisms

$$
h \circ \widehat{\phi}: \mathcal{S}^{-}(G ; C) \rightarrow C G \simeq C[A \times B] \rightarrow C
$$

is equal to the homomorphism $h_{\chi}$ for some character $\chi$ of $G$. Lemma 3.2 implies that $\pi_{\chi} \psi(s)=h_{\chi}(s)$. Since $h_{\chi}(s) \neq 0$, we get $\psi(s) \neq 0$. This implies that $\psi$ is a monomorphism. By the remark preceding Lemma 3.2, $\psi$ is also an epimorphism.

Corollary 3.4. For any $x, y \in S^{-}(G ; C), x \neq y$, there exists a character $\chi \in X(G)$ such that $h_{\chi}(x) \neq h_{\chi}(y)$.

Pr o of. If $x \neq y$ then $\psi(x) \neq \psi(y)$ in $C[X(G)]$. Therefore there exists a character $\chi \in$ $X(G)$ such that $\pi_{\chi}(\psi(x)) \neq \pi_{\chi}(\psi(y))$. Hence, Lemma 3.2 implies that $h_{\chi}(x) \neq h_{\chi}(y)$.

Added in proof (January 1998). In [P-S-2], we prove Theorem 3.1 for all finitely generated groups.

\section{References}

[B-1] D. Bullock, Estimating a skein module with $S L_{2}(C)$ characters, Proc. Amer. Math. Soc. 125 (1997), 1835-1839.

[B-2] D. Bullock, Estimating the states of the Kauffman bracket skein module, this volume.

[B-P] D. Bullock, J. H. Przytycki, Kauffman bracket quantization of symmetric algebra and $s o(3)$, preprint 1996.

[C-S] M. Culler and P. B. Shalen, Varieties of group representations and splittings of 3-manifolds, Ann. of Math. 117 (1983), 109-146.

[F-K] R. Fricke, F. Klein, Vorlesungen über die Theorie der automorphen Functionen, Vol. 1, pp. 365-370. Leipzig: B.G. Teubner 1897. Reprint: New York, Johnson reprint Corporation (Academic Press) 1965.

[Ho] R. D. Horowitz, Characters of free groups represented in two-dimensional special linear group, Comm. Pure and Appl. Math. 25, 1972, 635-649.

[H-P-1] J. Hoste, J. H. Przytycki, A survey of skein modules of 3-manifolds; in Knots 90, Proceedings of the International Conference on Knot Theory and Related Topics, Osaka (Japan), August 15-19, 1990, Editor A. Kawauchi, Walter de Gruyter 1992, 363-379

[H-P-2] J. Hoste, J. H. Przytycki, The $(2, \infty)$-skein module of lens spaces; a generalization of the Jones polynomial, Journal of Knot Theory and Its Ramifications, 2(3), 1993, 321-333.

[H-P-3] J. Hoste, J. H. Przytycki, The Kauffman bracket skein module of $S^{1} \times S^{2}$, Math . Z., 220(1), 1995, 63-73.

\footnotetext{
${ }^{8}$ It means that the affine variety, $B$, is isomorphic to the quasi-variety $C^{* k}$.
} 
[Hu] T. W. Hungerford, Algebra, Graduate Texts in Mathematics, Springer-Verlag 1974.

[Jo] T. Jorgensen, Closed geodesics on Riemann surfaces, Proc. Amer. Math. Soc., 72(1), 1978, 140-142.

[L-M] M. Lustig, W. Metzler, Integral representations of $A u t F^{n}$ and presentation classes of groups, Contemporary Mathematics, Vol 44, 1985, 51-67 (in Combinatorial Methods in Topology and Algebraic Geometry, Ed. J.R.Harper, R.Mandelbaum, Proceedings of a conference in honor of Arthur M.Stone, Rochester 1982).

[Ma-1] W. Magnus, Rings of Fricke characters and automorphism groups of free groups, Math. Z., 170, 1980, 91-103.

[Ma-2] W. Magnus, The use of 2 by 2 matrices in combinatorial group theory. A survey, Resultate der Mathematik, Vol. 4, 1981, 171-722.

[Pr] J. H. Przytycki, Skein modules of 3-manifolds, Bull. Polish Acad. Science, 39(1-2), 1991, 91-100.

[P-S-1] J. H. Przytycki, A. S. Sikora, On Skein Algebras And $S l_{2}(C)$-Character Varieties, Topology, submitted.

[P-S-2] J. H. Przytycki, A. S. Sikora, in preparation.

[V] H. Vogt, Sur les invariants fondamentaux des équations différentielles linéaires du second ordre. Ann. Sci. Ecole Norm. Sup. (3) 6, Suppl. 3-72 (1889) (Thèse, Paris). 